\title{
CASOS DE TUBERCULOSE EM POPULAÇÃO EM SITUAÇÃO DE RUA NO ESTADO DO RIO GRANDE DO NORTE: ANÁLISE DE DADOS SECUNDÁRIOS
}

\author{
CASES OF TUBERCULOSIS IN A STREET POPULATION IN RIO \\ GRANDE DO NORTE STATE: ANALYSIS OF SECONDARY DATA
}

Camila Priscila Abdias do Nascimento ${ }^{1}$ Rayla Patrícia da Silva Andrade Soares ${ }^{2}$

Rayane Saraiva Felix ${ }^{3}$

Sandy Yasmine Bezerra e Silva ${ }^{4}$ Érika Simone Galvão Pinto ${ }^{5}$

RESUMO: OBJETIVO: analisar os casos confirmados de tuberculose na população em situação de rua no estado do Rio Grande do Norte. MÉTODO: trata-se de estudo exploratório, quantitativo que analisou os dados de notificação de Tuberculose em população em situação de rua, no período 2014 a 2018. A pesquisa foi realizada com base nas informações estatísticas disponíveis no portal do Sistema de Informação de Agravos de Notificação. A coleta das informações foi realizada em dezembro de 2019 e as variáveis do estudo versam sobre: sexo, faixa, escolaridade, raça/cor e as regiões de saúde de confirmação de tuberculose em População em situação de rua. RESULTADOS: no período de 2014 a 2018 foram confirmados 166 casos de tuberculose em população em situação de rua no estado, $81,33 \%$ dos casos eram do sexo masculino com concentração de casos na faixa etária de 20 a 29 anos, $22,8 \%$ tem o ensino fundamental incompleto e a região metropolitana concentra 125 totais de caso de TB em PSR. CONCLUSÃO: a pesquisa permitiu identificar que a maior parte dos casos confirmados de tuberculose na população em situação de rua foram notificados em 2017, são do sexo masculino, com concentração na faixa etária 20 a 29 anos, possuem ensino fundamental incompleto

\footnotetext{
1 Enfermeira. Mestranda pelo Programa de Pós-Graduação em Enfermagem (PPGENF) da Universidade Federal do Rio Grande do Norte- UFRN, camilapriabd@gmail.com;

2 Enfermeira. Mestranda pelo Programa de Pós-Graduação em Enfermagem (PPGENF) da Universidade Federal do Rio Grande do Norte- UFRN, raylapatriciayla@gmail.com;

3 Enfermeira. Mestranda pelo Programa de Pós-Graduação em Enfermagem (PPGENF) da Universidade Federal do Rio Grande do Norte- UFRN, rsf1601@hotmail.com;

${ }^{4}$ Enfermeira. Doutoranda em Enfermagem pelo Programa de Pós-Graduação em Enfermagem (PPGENF) da Universidade Federal do Rio Grande do Norte - UFRN, sandyyasmine@hotmail.com;

${ }^{5}$ Enfermeira. Prof. ${ }^{a}$ Dra. do Programa de Pós-Graduação do Mestrado Acadêmico em Enfermagem da Universidade Federal do Rio Grande do Norte - UFRN, erikasgp@gmail.com.
} 
e se concentram na região metropolitana do estado. Esses achados podem contribuir para o desenvolvimento de ações específicas voltadas para esse público pelos profissionais da equipe de saúde e gestores.

Palavras chave: Tuberculose; Pessoas em Situação de Rua; Sistemas de Informação.

ABSTRACT: OBJECTIVE: To analyze the confirmed cases of tuberculosis in the homeless population in the state of Rio Grande do Norte. METHOD: This is an exploratory, quantitative study that analyzed tuberculosis notification data in homeless population from 2014 to 2018. The research was conducted based on the statistical information available on the website of the Disease Information System. Notification. The information was collected in December 2019 and the study variables are: gender, age, education, race / color and the health regions of confirmation of tuberculosis in homeless population. RESULTS: from 2014 to 2018 were confirmed 166 cases of tuberculosis in homeless population in the state, $81.33 \%$ of cases were male with concentration of cases in the age group of 20 to 29 years, $22.8 \%$ has incomplete primary education and the metropolitan region concentrates 125 TB case totals in PRS. CONCLUSION: the research identified that most confirmed cases of tuberculosis in the homeless population were reported in 2017, are male, with concentration in the age group 20 to 29 years, have incomplete elementary school and are concentrated in the region. metropolitan area. These findings may contribute to the development of specific actions aimed at this public by health team professionals and managers.

Keywords: Tuberculosis; People in Street Situation; Information systems. 


\section{INTRODUÇÃO}

A tuberculose (TB) é uma doença infectocontagiosa transmitida por via aérea, a partir da inalação de gotículas contendo os bacilos de Mycobacterium tuberculosis expelidos pela tosse, fala ou espirro do doente. A apresentação mais frequente da doença é a pulmonar, de grande relevância para a saúde pública, pois é a forma pulmonar bacilífera a responsável pela manutenção da cadeia de transmissão da doença (ROSSETTI, 2018).

O Brasil situando-se entre os 22 países que concentram $80 \%$ dos casos no mundo e ocupa a $17^{a}$ posição em relação ao total de números de casos. Em 2018, o país diagnosticou 72.788 casos novos de TB, perfazendo um coeficiente de incidência de 34,8/100.000 habitantes (BRASIL, 2019).

Diante do cenário, o Ministério da Saúde (MS) definiu algumas populações como prioritárias que demandam maior cuidado à saúde. Os grupos de população indígena, privados de liberdade, pessoas com Vírus da Imunodeficiência Humanal Síndrome da Imunodeficiência Adquirida (HIV/aids) e a População em Situação de Rua (PSR).

A PSR apresenta 56 vezes mais chances desenvolver a TB se comparada a população em geral em virtude da vulnerabilidade pelas condições que se encontram como situação de pobreza ou pobreza extrema (ARROYO et al, 2017).

Em 2016 um estudo realizado pelo Instituto de Pesquisa Econômica Aplicada (Ipea) estima cerca 102 mil pessoas em situação de rua no Brasil ocupando calçadas, viadutos e praças extremamente vulnerável ao adoecimento.

Pela fácil disseminação da TB o MS tem como estratégia para o enfrentamento dessa doença a deteç̧ão precoce para tratamento oportuno interrompendo a cadeia transmissora (Brasil, 2012).

Nos anos de 2014 a 2018 foram confirmados no Brasil 14.098 casos de TB na PSR, a região Sudeste apresenta o total de 7.897 casos de TB, seguido da região 
Sul com 2.638 casos, em seguida a região do Nordeste com 2.295 casos, o Norte com 686 casos e o Centro-Oeste com 582 casos de TB em PSR (BRASIL, 2019).

Esses dados estão disponíveis no Sistema de Informação de Agravos de Notificação (SINAN) por meio do Portal do DATASUS. O SINAN é responsável pelos registros de dados estatísticos de agravos em todo território nacional, alimentado pelas fichas de notificação compulsória, implantado gradualmente a partir de 1993 foi se desenvolvendo com intuito de obter o melhor gerenciamento de dados, atualmente visa o planejamento de políticas públicas de saúde além de realizar o acompanhamento constante das morbimortalidades.

No contexto das a PSR, estudo realizado na cidade do Rio de Janeiro identificou complexidade em relação a assistência à saúde e serviços de atenção relacionadas à fragmentação e precária intersetorialidade (ZUIM; TRAJMAN, 2018).

Uma das formas de implementar a assistência à saúde dessa população é identificar, analisar para que decisões possam ser planejadas e tomadas, tanto pela equipe de saúde que prestam assistência como pelos gestores.

Assim, esse trabalho tem como objetivo analisar os casos confirmados de TB em população em situação de rua no estado do Rio Grande do Norte.

\section{MÉTODO}

Trata-se de um estudo exploratório, de abordagem quantitativa.

Foram analisados os dados de notificação de TB em população em situação de rua do Brasil, no período 2014 a 2018. A pesquisa foi realizada com base nas informações estatísticas disponíveis no portal do SINAN. A escolha do ano de 2014 aconteceu por ter sido incluída a variável de PSR no SINAN-Net.

A coleta de dados ocorreu em dezembro de 2019 e foram utilizados os descritores "Tuberculose", "Pessoas em Situação de Rua" e "Sistemas de Informação" os quais estão indexados no Descritores em Ciência da Saúde (Decs).

As variáveis de interesses utilizadas neste estudo foram as características sociodemográficas como: sexo (masculino e feminino), faixa etária ( $<1$ ano, 15-19 
anos, 20-39 anos, 40-59 anos, 60-64 anos, 65-69 anos), escolaridade (analfabeto, fundamental incompleto, fundamental completo, ensino médio incompleto, ensino médio completo), raça/cor (branca, preta, amarela e parda) e as regiões de saúde. Os dados foram analisados através de estatística descritiva.

Por ser tratar de pesquisa com dados públicos de fonte secundária o estudo não foi submetido ao Comitê de Ética em Pesquisa.

\section{RESULTADOS}

No período de 2014 a 2018 foram confirmados 166 casos de TB em população em situação de rua no RN pelo SINAN, observou-se $28,31 \%$ (47 casos) foram confirmados no ano de 2017, conforme a tabela 1, entretanto em 2014 $3,03 \%$ (5 casos) foram notificados, os períodos analisados apresentam oscilação em virtude a notificação da PSR ter iniciado em 2014.

Os casos confirmados de TB na PSR no período de 2014 a 2018 no RN estão apresentados na tabela 1 abaixo:

Tabela 1 - Casos confirmados de Tuberculose em População em Situação de Rua no período de 2014 a 2018 no estado do Rio Grande do Norte.

\begin{tabular}{ccc}
\hline $\begin{array}{c}\text { Ano de casos confirmados de TB em } \\
\text { PSR }\end{array}$ & $\begin{array}{c}\text { Total casos confirmados de TB em } \\
\text { PSR }\end{array}$ \\
& $\mathbf{n ~ ( 1 6 6 )}$ & $\%$ \\
\hline 2014 & 5 & $3,03 \%$ \\
\hline 2015 & 45 & $27,10 \%$ \\
\hline 2016 & 34 & $20,48 \%$ \\
\hline 2017 & 47 & $28,31 \%$ \\
\hline 2018 & 35 & $21,08 \%$ \\
\hline
\end{tabular}

Fonte: SINAN.

Entre os casos registrados, $135(81,33 \%)$ eram do sexo masculino e $31(18,67 \%)$ femininos, em relação a faixa etária observa-se uma concentração na 
faixa etária 20 a 29 anos (56,02\%) seguido da faixa etária 40 a 59 anos (36,74\%), como ilustra a tabela 2.

Tabela 2 - Faixa etária da População em Situação de Rua com Tuberculose confirmada no período de 2014 a 2018 no estado do Rio Grande do Norte.

\begin{tabular}{cc}
\hline Faixa etária & $\mathbf{n ~ ( 1 6 6 )}$ \\
\hline$<1$ ano de idade & 1 \\
\hline $15-19$ anos de idade & 4 \\
\hline $20-39$ anos de idade & 93 \\
\hline $40-59$ anos de idade & 61 \\
\hline $60-69$ anos de idade & 7 \\
\hline
\end{tabular}

Fonte: SINAN.

Tratando-se do nível de escolaridade 50 (30,12\%) casos foi ignorado, a variável referente a essa informação não foi preenchida, $38(22,8 \%)$ tinham o ensino fundamental incompleto, os analfabetos representavam $28(16,86 \%)$ e nenhum dos pacientes do estudo tinham ensino superior.

Referente a raça/cor pardos são a maioria 123 (74,09\%), seguidos de brancos $17(10,24 \%)$, preta $16(9,63 \%)$, amarela $1(0,62 \%)$ e ignorado $9(5,42 \%)$.

As regiões de saúde por residência apresentadas na tabela 3 retrata os casos de TB em população em situação de rua no estado do RN como maior concentração de casos a região metropolitana com 125 dos 166 casos total, a capital Natal reúne 120 casos. 
Tabela 3 - Regiões de Saúde por Residência da População em Situação de Rua com TB no período de 2014 a 2018 no estado do Rio Grande do Norte.

\begin{tabular}{cc}
\hline Regiões de Saúde & $\mathbf{n ~ ( 1 6 6 )}$ \\
\hline São José do Mipibu & 5 \\
\hline Mossoró & 18 \\
\hline João Câmara & 3 \\
\hline Caicó & 1 \\
\hline Santa Cruz & 6 \\
\hline Pau dos Ferros & 2 \\
\hline Metropolitana & 125 \\
\hline Açu & 6 \\
\hline
\end{tabular}

Fonte: SINAN.

\section{DISCUSSÃO}

A análise dos dados permitiu verificar que o ano de 2017 obteve o maior número de casos confirmados $(28,31 \%)$ de TB na PSR e o menor número de casos foi no ano de 2014 (3,03\%). Esses resultados devem-se ao fato de que em 2014 foi inserida a variável de PSR no SINAN-Net. O crescimento no número de casos notificados no decorrer dos anos pode indicar um maior monitoramento em relação a essa população, com a busca ativa de sintomáticos respiratórios.

Em relação a sexo, a maioria dos casos na PSR era do sexo masculino. Um estudo realizado em São Paulo em 2015 constatou que 82\% dos moradores de rua eram do sexo masculino e apresentavam idades entre 18 a 49 anos (INEP, 2015).

Assim diante do estudo a faixa etária assemelham-se da médica nacional na PSR.

A respeito do nível de escolaridade, em $30,12 \%$ dos casos essa variável não foi preenchida o que se torna preocupante pois indica uma falha no preenchimento das informações. Das informações disponíveis, $22,8 \%$ dos casos tinham o ensino fundamental incompleto e nenhum dos pacientes do estudo tinham ensino superior. 
Sabe-se que uma doença de cunho social como a TB, necessita de que a educação em saúde seja feita em função de empoderar cidadãos adoecidos, de modo que, além do tratamento e acesso aos serviços de saúde, possam reivindicar melhoria de qualidade de vida (SÀ et al, 2013).

Quanto às regiões de saúde por residência, os casos de TB em PSR tem maior concentração de casos na região metropolitana $(75,30 \%)$. A capital Natal reúne (72,28\%) 120 casos. Um estudo realizado em Niterói-RJ apontou que os bairros da região que compõem o município apresentam grandes riscos para produção da TB devido a relação da manutenção da endemia com as condições de vida no grupo populacional vulnerável (VALENTE et al, 2019).

\section{CONCLUSÃO}

A presente pesquisa permitiu identificar que a maior parte dos casos confirmados de tuberculose na população em situação de rua foram notificados em 2017, são do sexo masculino, com concentração na faixa etária 20 a 29 anos, possuem ensino fundamental incompleto e se concentram na região metropolitana do estado.

O presente estudo corrobora que a TB em PSR se caracteriza como um problema de saúde pública que deve estimular uma mobilização sociopolítica.

Espera-se que esses achados possam contribuir para o desenvolvimento de ações específicas voltadas para esse público pelos profissionais da equipe de saúde e gestores.

Como limitação do estudo elenca-se a necessidade de estudos locais e mais profundos, afim de evidenciar as características da TB na PSR. 


\section{REFERÊNCIAS BIBLIOGRÁFICAS}

ARROYO, L. H, et al. Identificação de áreas de risco para a transmissão da tuberculose no município de São Carlos, São Paulo, 2008 a 2013. Epidemiol. Serv. Saúde 26 (3) Jul- Sep 2017 Disponível em: https://doi.org/10.5123/S167949742017000300010https://www.scielosp.org/scielo.php?pid=S 223796222017000300525\&script=sci_arttext\&tIng=pt\#.

BRASIL. Ministério da Saúde. Secretaria de Vigilância em Saúde. Boletim Epidemiológico, v. 50, n. 9, 2019. Indicadores prioritários para o monitoramento do Plano Nacional pelo Fim da Tuberculose como Problema de Saúde Pública no Brasil. Brasília: Ministério da Saúde, 2019.

SINAN. Sistema de Informação de Agravos de Notificação. Disponível em http://dtr2004.saude.gov.br/sinanweb/ Acesso em dez. 2019.

BRASIL. Ministério da Saúde. Secretaria de Atenção à Saúde. Departamento de Atenção Básica. Manual sobre o cuidado à saúde junto a população em situação de rua. Brasília: Ministério da Saúde; 2012.

INEP. Fundação e Instituto de Pesquisas Econômicas (BR). Secretaria de Assistência e Desenvolvimento Social. Prefeitura de São Paulo. Censo da População em Situação de Rua da Cidade de São Paulo. Fundação e Instituto de Pesquisas Econômicas. Secretaria de Assistência e Desenvolvimento Social. Prefeitura de São Paulo, São Paulo: Fipe/SMADS; 2015. Disponível em: http://www.prefeitura.sp.gov.br/cidade/secretarias/upload/assistencia_social/observatorio_soc ial/2015/censo/FIPE_smads_CENSO_2015_coletivafinal.pdf.

ROSSETTI M.L.R, et al. Novas tecnologias para estudo da tuberculose: Uma análise da detecção e transmissão de $M$. tuberculosis circulante. Comunicação em Ciências da Saúde, [S.I.], v. 28, n. 01, p. 85-90, feb. 2018. ISSN 1980-5101. Disponível em: http://www.escs.edu.br/revistaccs/index.php/comunicacaoemcienciasdasaude/article/view/1 24 .

SÁ, L. D. et al. Educação em saúde no controle da tuberculose: perspectiva de profissionais da estratégia Saúde da Família. Rev. Eletr. Enf. [Internet]. 2013 jan/mar;15(1):103-11.

Disponível

em:

http://dx.doi.org/10.5216/ree.v15i1.15246.https://www.fen.ufg.br/revista/v15/n1/pdf/v15n1a12.pdf.

VALENTE, B. C et al. A tuberculose e seus fatores associados em um município da região metropolitana do Rio de Janeiro. Rev. bras. epidemiol., São Paulo, v. 22, e190027,

2019. Disponível em: http://www.scielo.br/scielo.php?script=sci_arttext\&pid=S1415790X2019000100425\&lng=en\&nrm=iso. Acesso em: 08 jan. 2020. http://dx.doi.org/10.1590/1980-549720190027.

ZUIM, Regina Célia Brazolino; TRAJMAN, Anete. Itinerário terapêutico de doentes com tuberculose vivendo em situação de rua no Rio de Janeiro. Physis, Rio de Janeiro, v. 28, n. 2, e280-205, $2018 . \quad$ Disponível em: http://www.scielo.br/scielo.php?script=sci_arttext\&pid=S010373312018000200601\&lng=pt \&nrm=is. Acesso em: 08 jan. 2020. 